\title{
Plastic and Reconstructive Surgery
}

\section{Non-activated versus thrombin-activated platelets on wound healing and fibroblast-to- myofibroblast differentiation in vivo and in vitro \\ --Manuscript Draft--}

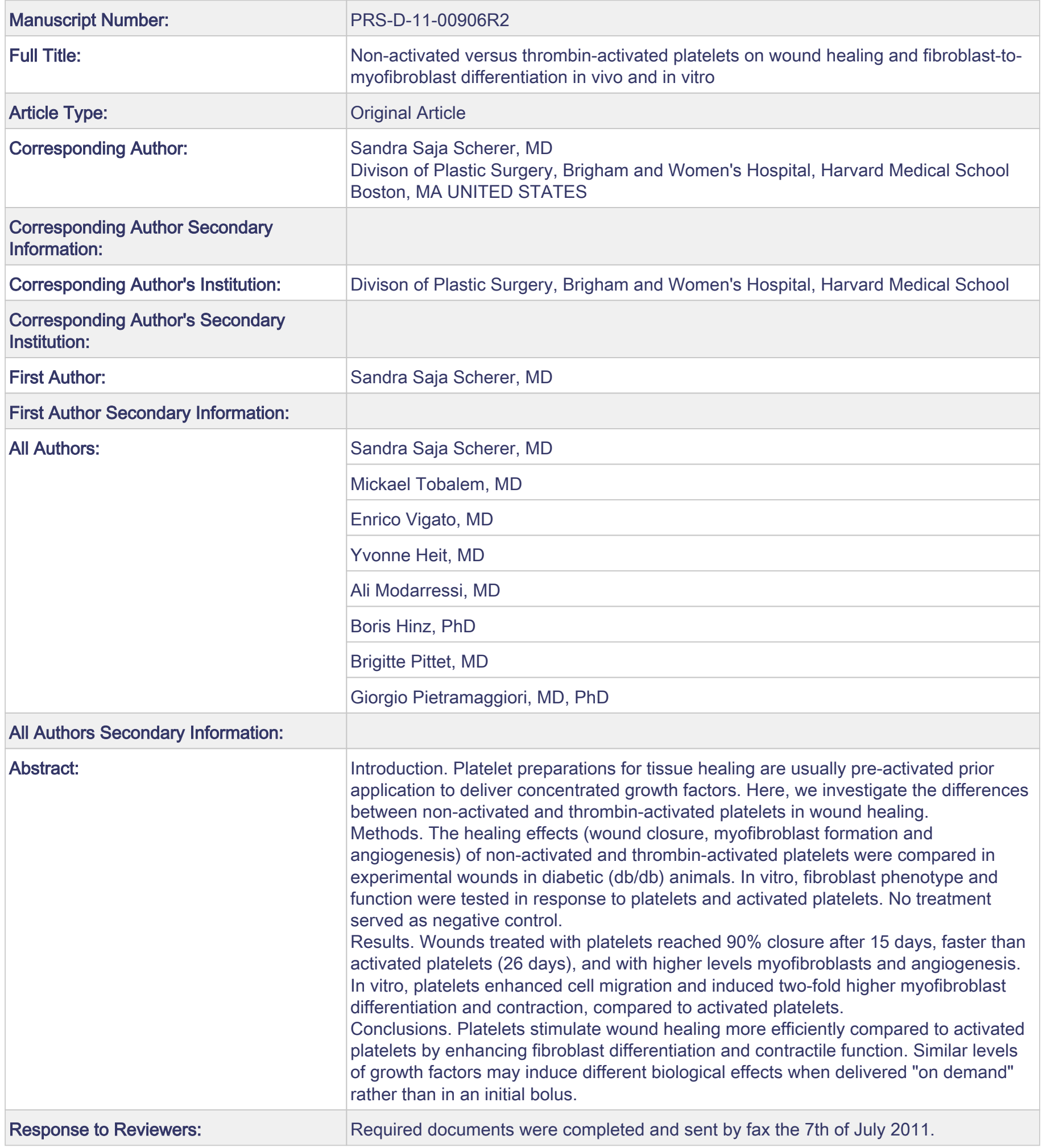




\section{Non-activated versus thrombin-activated platelets on wound healing and fibroblast-to-} myofibroblast differentiation in vivo and in vitro

Sandra S. Scherer ${ }^{1}$, Mickael Tobalem ${ }^{2}$, Enrico Vigato ${ }^{2,3}$, Yvonne Heit ${ }^{4}$, Ali Modarressi ${ }^{2}$, Boris Hinz ${ }^{5}$, Brigitte Pittet $^{2}$, and Giorgio Pietramaggiori ${ }^{1,2,3}$

${ }^{1}$ Department of Plastic Surgery, University Hospitals of Lausanne, Lausanne, Switzerland, ${ }^{2}$ Department of Plastic, Reconstructive \& Aesthetic Surgery, University Hospitals of Geneva and Faculty of Medicine, University of Geneva, Geneva, Switzerland, ${ }^{3}$ Laboratory of Cell Biophysics, Ecole Polytechnique Fédérale de Lausanne, Lausanne, Switzerland, ${ }^{4}$ Tissue Engineering \& Wound Healing Laboratory, Brigham and Women's Hospital, Boston, USA, ${ }^{5}$ Laboratory of Tissue Repair and Regeneration, Matrix Dynamics Group, Faculty of Dentistry, University of Toronto, Toronto, Canada

\section{Corresponding Authors:}

Giorgio Pietramaggiori, $\mathrm{MD}, \mathrm{PhD}$

Service de chirurgie plastique et reconstructive

Département de Chirurgie

Centre Hospitalier Universitaire Vaudois

Rue du Bugnon 46

1011 Lausanne

gpietramaggiori@gmail.com
Brigitte Pittet, MD

Service de chirurgie plastique et reconstructive et esthétique

Département de Chirurgie

Hôpitaux Universitaires de Genève

Gabrielle-Perret-Gentil 4

1211 Genève 14

brigitte.pittet-cuenod@hcuge.ch

Running Title: Platelets: cell therapy for wound healing

Key words: platelet, thrombin, angiogenesis, wound healing, wound contraction, myofibroblasts, cell contraction, cell therapy 
Financial Disclosure: None of the authors has a financial interest in any of the products, services, or drugs mentioned in this manuscript. 


\section{ABSTRACT}

Introduction. Platelet preparations for tissue healing are usually pre-activated prior application to deliver concentrated growth factors. Here, we investigate the differences between non-activated and thrombin-activated platelets in wound healing.

Methods. The healing effects (wound closure, myofibroblast formation and angiogenesis) of non-activated and thrombin-activated platelets were compared in experimental wounds in diabetic $(\mathrm{db} / \mathrm{db})$ animals. In vitro, fibroblast phenotype and function were tested in response to platelets and activated platelets. No treatment served as negative control.

Results. Wounds treated with platelets reached $90 \%$ closure after 15 days, faster than activated platelets (26 days), and with higher levels myofibroblasts and angiogenesis. In vitro, platelets enhanced cell migration and induced two-fold higher myofibroblast differentiation and contraction, compared to activated platelets.

Conclusions. Platelets stimulate wound healing more efficiently compared to activated platelets by enhancing fibroblast differentiation and contractile function. Similar levels of growth factors may induce different biological effects when delivered "on demand" rather than in an initial bolus. 


\section{INTRODUCTION}

With the increasing prevalence of diabetes and obesity over the last few decades, a concurrent surge in chronic wounds and complex tissue defects has been registered. ${ }^{1}$ Among the different treatments that have been developed to improve healing, topical application of autologous platelets offered the advantage to promptly provide high doses of growth factors without adding significant costs.

Platelets have been the object of clinical and experimental wound healing studies for more than 30 years. $^{2}$ Recent discoveries in basic science revealed that platelets are not only playing a key role in hemostasis, but participate actively in multiple biological events such as tissue regeneration, cell proliferation and immunological defense. ${ }^{3}$ During the early phases in wound healing platelets participate in initiating repair by forming a provisional fibrin scaffold that allows cell migration into the tissue defects. In later phases, platelets support the recruitment, differentiation and cross talk of cells by releasing several bioactive soluble factors. ${ }^{4,5}$ Most likely, during physiological wound healing, platelets participate in these events in a timecontrolled fashion.

In clinical practice, surgeons commonly use activated platelets by pre-treatment with thrombin and/or calcium to induce the immediate and full release of soluble factors. It has been shown that upon thrombin activation, platelets release most of their content, inducing hundred folds increases in growth factor concentrations in plasma over non-activated controls. ${ }^{6,7}$ However, once activated, platelets lose the ability to specifically interact with the environment. ${ }^{8,9}$ For example, the structural and functional integrity of platelets is required to promote angiogenesis, whereas platelet derived growth factors such as vascular endothelial 
growth factor (VEGF) alone are less efficient. ${ }^{10}$ Consistently, treatment with growth factors in high doses has met only limited success in clinical applications. ${ }^{11}$

We hypothesize that non-activated platelets are more efficient to stimulate wound healing than thrombin pre-activated ones. We propose that platelets may be used as a cell therapy rather than exploited only for their growth factor content in wound healing. To explore this hypothesis, we compared the effects of activated versus non-activated platelets in vivo in a diabetic wound healing model and in vitro on fibroblasts.

\section{MATERIALS AND METHODS}

\section{Preparation of platelets}

Single donor human blood was collected into 1:10 v/v sodium citrate and placed on a Nutator Mixer (BD Diagnostics) until processed. Platelets in plasma were obtained by centrifugation of the citrated blood at 180-times $g$ for $20 \mathrm{~min}$ at room temperature, followed by collection of the upper phase. The platelet concentration was then adjusted to $3 \times 10^{5} / \mu 1$ in the same plasma. Platelets were stored at room temperature $\left(22^{\circ} \mathrm{C}\right)$ on an Eberbach shaker until used. In blinded experiments platelets were applied platelets either non-activated or after activation with 100 UI thrombin per $\mathrm{ml}$ immediately before application.

\section{In vivo wound model}

We used 8-12 week-old homozygous genetically diabetic $\mathrm{db} / \mathrm{db}$ male mice (strain C57BL/KsJ-Lepr ${ }^{\mathrm{db}}$ ) as accepted model for impaired wound healing. ${ }^{12}$ Animals were used under an approved animal protocol in an accredited facility. The day prior to surgery, hair was clipped and depilated (Nair ${ }^{\circledR}$, Church \& Dwight Co., Princeton, NJ). On the day of the surgery, animals were weighted and anesthetized with $60 \mathrm{mg} / \mathrm{kg}$ Nembutal (Pentobarbital). A 
dorsal $1.0 \mathrm{~cm}^{2}$ area of skin, including the panniculus carnosus was excised. Wounds were then photographed and individually sealed with semi-occlusive polyurethane dressings (Tegaderm $^{\mathrm{TM}}, 3 \mathrm{M}$, St. Paul, MN) that was kept in place between dressing changes. Injection of $100 \mu 1$ of platelet preparations using a 30 gauge needle through the dressing into randomly selected wounds followed ( $\mathrm{n}=12 /$ treatment group). Preparations of activated or non-activated platelets were administered once on the day of the surgery or wounds were left untreated in the control group (NT).

\section{Wound closure analysis}

To macroscopically assess wound closure, digital photographs were captured twice a week and compared with initial photographs by two independent treatment-blinded observers using planimetric methods (Image J, NIH, Bethesda, MD). Wound closure was quantified as percentage of the original wound area and expressed as wound contraction and reepithelialization. The sum of contracted, re-epithelialized, and open wound areas equals $100 \%$ of the original wound size, as previously described. ${ }^{13}$

For histological and immunohistochemical analysis, six animals were sacrificed per group 7 days post-wounding and excised wounds were fixed in $4 \%$ buffered formaldehyde. Central wound cross-sections were embedded in paraffin, sectioned and stained according to routine hematoxylin and eosin $(\mathrm{H} \& \mathrm{E})$ protocols. Panoramic cross-sectional digital images of each wound were prepared using Adobe Photoshop. Sections were analyzed with digital planimetry (Image J, NIH, Bethesda, MD) by two independent treatment-blinded observers.

\section{Antibodies and microscopy}


For immunofluorescence we used primary antibodies against the myofibroblast marker $\alpha$ smooth muscle actin ( $\alpha$-SMA) (mouse IgG2a, $\alpha$ SM-1, a gift from Dr. G. Gabbiani, University of Geneva, CH). ${ }^{14}$ For cell culture experiments, primary antibodies were added after cell fixation and permeabilization, and probed with AlexaFluor-conjugated goat anti-mouse and goat anti-rabbit secondary antibodies (Molecular Probes, Invitrogen, Basel, CH). DNA was probed with DAPI (Sigma) and F-actin with Phalloidin-Alexa 488 (Molecular Probes). Phase contrast and epifluorescence microscopy was performed using oil immersion objectives (PlanNeofluar 40x/1.2 Ph3, Plan-Neofluar 63x/1.4 Ph3, Zeiss) mounted on an inverted microscope (Axiovert 135, Carl Zeiss AG, Feldbach, CH) and digital CCD camera (Hamamatsu C474295-12ERG, Bucher Biotec AG, Basel, CH). Images were acquired with Openlab 3.1.2 software (Improvision, Basel, $\mathrm{CH}$ ) and assembled with Adobe Photoshop CS3 (Adobe Systems Incorporated, San Jose, CA).

For immunohistochemistry of tissues, excised wounds were fixed in $4 \%$ buffered formaldehyde. Immunostaining for $\alpha$-SMA was performed on paraffin-embedded transverse sections using biotinylated goat anti-mouse IgG2a (Jackson Immunoresearch, West Grove, PA) and treatment with streptavidin-biotin-peroxidase (Dako, Glostrup, Denmark). Peroxidase activity was detected with diaminobenzidine (Serva, Heidelberg, Germany), as previously described. ${ }^{15}$ Software aided quantification of $\alpha$-SMA positive cells and stress fibers was performed as previously described. ${ }^{15}$ To evaluate $\alpha$-SMA expression as an indicator of the presence of myofibroblasts, vessels were manually excluded from the image to retain only the area of interest.

For all other stainings, paraffin-embedded sections were re-hydrated and treated with 40 $\mu \mathrm{g} / \mathrm{ml}$ proteinase $\mathrm{K}$ (Roche Diagnostics Corp.) for $25 \mathrm{~min}$ at $37^{\circ} \mathrm{C}$ as previously described. ${ }^{16}$ 
Primary antibodies against PECAM-1 (Pharmingen, San Jose, CA) were incubated at $4{ }^{\circ} \mathrm{C}$ overnight. PECAM-1 signal was intensified using the tyramide amplification system (PerkinElmer, Boston, MA). The total number of blood vessels (positive for PECAM-1) was counted using 40x magnification for three pictures (one from the centre and two from the margins of the wound) for each sample. Six samples were used for each condition and epitope.

\section{Cell culture, and cell contraction assays}

Primary human dermal fibroblasts, obtained from three different healthy donors, were grown from dermal explants and used between passages 2 to 5 as previously described. ${ }^{17}$ Cells were seeded onto collagen coated (collagen type I, $10 \mu \mathrm{g} / \mathrm{ml}$, Sigma) glass coverslips at 5'000 cells $/ \mathrm{cm}^{2}$ Experiments were performed with fibroblasts from at least three different human samples from discarded tissues as by an approved ethical protocol of our hospital. Fibroblast cultures were treated on the first day with $3 \times 10^{5}$ activated or non-activated platelets per $\mathrm{ml}$ of media and compared to control medium (DMEM (Gibco-Invitrogen Basel, Switzerland) with $20 \%$ fetal calf serum (HyClone, Thermo-Fisher Scientific, Waltham, MA) for 1 to 5 days. Platelet preparations were added on the collagen coated surface prior to cells and left to incubate 15 minutes with the fibroblasts before media addition.

We observed that fibroblasts formed membrane extensions shortly after contact with platelets that delimitated vessel like structures. The number of cells contributing to confluent structures was counted per high power field in three representative areas. Wrinkling silicone substrates (Excellness Biotech SA, Lausanne, Switzerland) were used to identify individual contractile cells. ${ }^{18}$ Substrates were used with a stiffness of $10 \mathrm{kPa}$ to restrict wrinkle formation to highly contractile $\alpha$-SMA-positive cells. Substrates were rendered cell-adhesive with collagen type I (10 $\mu \mathrm{g} / \mathrm{ml}$, Sigma) and stiffness was controlled by atomic force microscopy (Nanowizard II, 
JPK instruments, Berlin, Germany). ${ }^{19}$ As another contraction test, fibroblasts were grown in attached three-dimensional collagen lattices. ${ }^{15}$ Populations were initiated with $0.25-10.0 \times 10^{5}$ cells $/ \mathrm{ml}$ collagen type I $(1.0 \mathrm{mg} / \mathrm{ml})$ (Sigma) adapted to obtain similar cell numbers at the time of contraction measurement after 24 hours of growth.

\section{In vitro wound healing assay}

To characterize the interactions between platelet preparations and fibroblasts in an in vitro wound healing assay, modified fibroblast-populated collagen gels $\left(1.75 \times 10^{5}\right.$ cells $\left./ \mathrm{ml}\right)$ were prepared with a circular hole in the centre to simulate a tissue defect. The hole was created by placing a $5 \mathrm{~mm}$ diameter tube in the middle of a $35 \mathrm{~mm}$ culture dish while adding $400 \mu \mathrm{l}$ of collagen solution in acetic acid. The tube was removed after gels were polymerized under neutralization with $\mathrm{NaOH}$. Activated or non-activated platelets $(100 \mu \mathrm{l})$ were then added to fill the hole and left incubate prior media addition for 15 minutes. Fibroblast growth into the tissue defect was morphologically assessed every day using contrast-phase microscopy for 21 days.

\section{Statistical analysis}

All values are expressed as mean \pm standard deviation. One-way analysis of variance and $a d$ hoc LSD variance analysis was used to determine the significance of differences between treatment modes. Multivariate analysis was performed using Statistica v7.0 (StatSoft, Inc, Tulsa, OK). A p-value of $<0.05$ was considered significant. 


\section{RESULTS}

\section{Application of platelets accelerates healing of diabetic mouse wounds}

To test the differences between activated and non-activated platelets on experimental wound healing, we generated full thickness skin defects on the dorsum of diabetic mice. Human platelets were used in order to obtain single donor samples with standardized levels of activation and cytokines. As previously published, single topical doses of human platelets applied to mouse wounds did not induce any aberrant immunological response. ${ }^{8}$ Single topical application of non-activated platelets improved wound contraction after 7 days (P, 40\%, $\mathrm{p}<0.05)$ compared to treatment with activated platelets $(\mathrm{aP}, 25 \%)$ and untreated wounds (NT, 15\%) (Fig. 1, Left Column). Both, activated and non-activated platelets induced similar levels of re-epithelialization (15\% and 8\%, respectively) (Fig. 1 Left Column, indicated by arrows), while NT wounds $(\mathrm{p}<0.05)$ showed only scarce signs of epithelium. In the later phases of wound healing, platelets significantly accelerated wound closure compared with activated platelets and non-treated wounds. Non-activated platelet treated wounds reached $90 \%$ wound closure, 1.7-times faster (day 15) compared to wounds treated with activated platelets (26 days, p<0.05, Fig. 1, Right Upper), and 2.3-times faster than NT wounds (35 days, p<0.01, Fig. 1, Right Upper). Wounds treated with activated platelets reached $90 \%$ wound closure 1.3-fold faster than NT wounds ( $\mathrm{p}<0.05$, Fig. 1, Right Upper).

Non-activated platelets induced higher expression of $\alpha$-SMA and angiogenesis in healing wounds than activated platelets

To test whether enhanced wound contraction is related to higher occurrence of contractile myofibroblasts, we assessed expression of $\alpha$-SMA in cross-sections of wounds on day 7. Wounds treated with activated and non-activated platelets both exhibited increased levels of $\alpha$-SMA expression (6-fold, $\mathrm{p}<0.001$ and 3.8-fold, $\mathrm{p}<0.05$, respectively) compared to NT 
wounds 7 days post-wounding (Fig. 1, Right Middle). Platelets significantly stimulated $\alpha-$ SMA expression by 1.5 -fold compared to activated platelets (Fig. 1, Right Middle, $\mathrm{p}<0.05$ ).

Blood vessel density was assessed by immunlocalization of PECAM-1 in the granulation tissue on day 7. The highest density (37.5 blood vessels/microscopic field) was measured in 7 day-old wounds treated with non-activated platelets $(\mathrm{p}<0.05$ compared to activated platelets, Fig. 1, Central Middle, Right Lower). Angiogenesis was 7.3-fold higher compared to NT ( $\mathrm{p}<0.001,5.1$ blood vessels/image field) and 3.7-fold higher compared to activated platelets wounds (10 blood vessels/image field, p<0.05) (Fig. 1, Central Column, Right Lower).

\section{Non-activated platelets induce an areolar organization of fibroblasts and stimulate expression of $\alpha$-SMA in vitro}

To understand the role of platelets in fibroblast to myofibroblast differentiation in wound healing, human dermal fibroblasts were cultured on two-dimensional collagen-coated glass coverslips in control media and in the presence of activated or non-activated platelets.

Comparable numbers of fibroblasts were quantified in control media and platelet treatments (106 and 88 cells/image field at 10x magnification respectively), which were found significantly higher $(\mathrm{p}<0.05)$ compared to activated platelets treatment $(42$ cells/image field $)$. In control media, fibroblasts grew separated and tended to become confluent over a period of 5 days with random spatial distribution. In the presence of activated platelets, fibroblasts developed non-organized dendritic appendages starting from 24h (Fig. 2, Upper Row), while non-activated platelet treatment induced a more organized blood vessel-like pattern, formed by the interdigitation of the dendritic appendages of neighboring cells (Fig. 2, Upper Row). Notably, already 24 hours after platelet treatment the formation of long dendritic, syncytial structures, interconnecting adjacent fibroblasts was noted (Fig. 2, Left Upper row and Right). 
These blood vessel-like structures were found to be 10-fold increased compared to control and activated platelet conditions $(\mathrm{p}<0.01)$.

The level of myofibroblast differentiation in culture was quantified by immunofluorescence staining for $\alpha$-SMA and cell counting. After 5 days of culture, non-activated platelets most efficiently induced myofibroblast differentiation with up to $57.3 \% \alpha$-SMA positive cells compared to control media $(21.5 \%)$ and activated platelet treatment $(29.6 \%$, Fig. 2, Left Middle row, $\mathrm{p}<0.01$ ). We further assessed the contractile function of fibroblasts by testing their ability to generate wrinkles on the surface of soft silicone culture substrates, 24 hours after treatment. Platelets induced a higher amount of wrinkling and contractile cells (35\%, $\mathrm{p}<0.05)$ compared to control media $(20 \%)$ or activated platelets $(10 \%$, Fig. 2, Left Lower row, wrinkles indicated by arrows).

\section{Non-activated platelets stimulate fibroblast migration into in vitro extracellular matrix}

\section{defects}

To finally test the effect of platelets on the capacity of fibroblasts to fill a "tissue defect", we prepared fibroblast-populated donut-shaped collagen gels (Fig. 3, Left). Activated or nonactivated platelets were then added to the fibroblast- and extracellular matrix-free central space. Activated platelets formed a clot (Fig. 3, Middle) where virtually no fibroblasts were found up to 21 days of culture (not shown). In contrast, when non-activated platelets were added, fibroblasts actively migrated into the centre (Fig. 3, Right) from the first day of culture. Fibroblasts that migrated into the centre after platelet treatment survived up to 21 days after seeding as tested by light microscopy (not shown). 


\section{DISCUSSION}

Platelets, commonly pre-activated, are widely used for non-healing wounds as a cost effective source of growth factors. ${ }^{4}$ Here, we first compared the effect of activated versus non-activated platelets in multiple experimental settings. Our in vivo and in vitro results show that thrombinactivation causes partial loss of the platelets healing properties. Platelets promoted better healing than activated platelets by stimulating 1) more efficient wound contraction and faster wound closure, 2) fibroblast migration and differentiation into contractile myofibroblasts, and 3) formation of capillary-like structures by fibroblasts in vitro, which corresponded to enhanced angiogenesis in vivo.

Blood vessels provide the infrastructure for nutrition and cell delivery to the wound. Angiogenesis occurs by the sprouting of endothelial cells into the new forming tissue. It is known that dermal fibroblasts regulate blood vessel formation through the expression of matrix metalloproteinases (e.g., MT-1 MMP) or other cytokines and growth factors (e.g. TIMP-1 and VEGF-C) in tumors and healing wounds. ${ }^{20,21,22}$ Here, we observe for the first time in vitro that interactions of platelets with fibroblasts, as early as 24 hours after culture, generate capillary-like structures in the absence of endothelial cells. It is possible that these structures formed by fibroblasts in the maturing extracellular matrix are the first event, which is necessary to physically guide endothelial cells to form new blood vessels. This view is supported by a sentinel function of fibroblastic cells played during tumor vascularization. ${ }^{23}$ Interestingly, these dendritic structures were characteristically $\alpha$-SMA negative, possibly to prevent the obliteration of the intravascular space by contraction before endothelial cell migration, in the early phases of angiogenesis. More studies are required to further investigate the importance of fibroblast and platelets as primary "angioarchitects" during normal wound healing. 
It is becoming increasingly evident that platelets have a function in wound healing going beyond their role in hemostasis. Recent fundamental work showed that anti-angiogenic factors like endostatin and pro-angiogenic factors like VEGF are both stored in platelets, but released independently and antagonistically upon specific receptor interaction (PAR4 for endostatin and PAR1 for VEGF). ${ }^{24,} 25$ Thrombin (factor II), the most commonly used factor to pre-activate platelets for wound healing, activates both receptors un-specifically, leading to a potential contemporary release of stimulatory and inhibiting growth factors at once. ${ }^{9,26}$ Since the growth factor content was standardized between activated and non-activated platelets (same quantity from single donor samples), the differences in the effect may be attributed to the way the growth factors were presented to the cells (Fig. 4). While activated platelets delivered all the growth factors (pro and anti angiogenic) in a concentrated bolus, nonactivated platelets likely released growth factors more specifically, upon receptor interactions. $^{6,27}$ The possibility to harvest platelet drug delivery ability for wound healing is a fascinating hypothesis that deserves to be further supported by additional mechanistic studies.

In the effort to best harvest the properties of platelet therapy for wound healing, activation is not the only variable that should be studied. Several other aspects should be considered such as the activation of platelet aggregation (calcium) or the protocol for platelets preparation (centrifugation and final concentration) and topical application (alone or with associated specific dressing).

In conclusion, our results support the use of non-activated physiologic platelets for wound healing, to best activate fibroblasts differentiation and migration, promote angiogenesis and 
ultimately stimulate healing. Future studies will concentrate on the characterization of growth factor delivery by non-activated platelets during wound healing.

\section{ACKNOLEDGEMENTS}

This research was supported by the Fond National Suisse grant \#310030_120571 (to BP and BH), by the Heart and Stroke Foundation Ontario (grant \#NA7086 to BH), and the Canadian Institutes of Health Research (grant \#210820 to BH). Authors thank Dr. Robert Valeri for useful discussion and critical review of the manuscript. 


\section{REFERENCES}

1. www.idf.org/home/index.cfm. Complications of Diabetes in the United States.

2. Knighton DR, Ciresi KF, Fiegel VD, et al. Classification and treatment of chronic nonhealing wounds. Successful treatment with autologous platelet-derived wound healing factors (PDWHF). Ann Surg 1986; 204(3):322-30.

3. Lesurtel M, Graf R, Aleil B, et al. Platelet-derived serotonin mediates liver regeneration. Science 2006; 312(5770):104-7.

4. Eppley BL, Pietrzak WS, Blanton M. Platelet-rich plasma: a review of biology and applications in plastic surgery. Plast Reconstr Surg 2006; 118(6):147e-159e.

5. Eppley BL, Woodell JE, Higgins J. Platelet quantification and growth factor analysis from platelet-rich plasma: implications for wound healing. Plast Reconstr Surg 2004; 114(6):1502-8.

6. Kakudo N, Minakata T, Mitsui T, et al. Proliferation-promoting effect of platelet-rich plasma on human adipose-derived stem cells and human dermal fibroblasts. Plast Reconstr Surg 2008; 122(5):1352-60.

7. Bambace NM, Levis JE, Holmes CE. The effect of P2Y-mediated platelet activation on the release of VEGF and endostatin from platelets. Platelets; 21(2):85-93.

8. Pietramaggiori G, Scherer SS, Mathews JC, et al. Quiescent platelets stimulate angiogenesis and diabetic wound repair. J Surg Res 2010; 160(1):169-77.

9. Ma L, Perini R, McKnight W, et al. Proteinase-activated receptors 1 and 4 counterregulate endostatin and VEGF release from human platelets. Proc Natl Acad Sci U S A 2005; 102(1):216-20.

10. Kisucka J, Butterfield CE, Duda DG, et al. Platelets and platelet adhesion support angiogenesis while preventing excessive hemorrhage. Proc Natl Acad Sci U S A 2006.

11. Chan RK, Liu PH, Pietramaggiori G, et al. Effect of recombinant platelet-derived growth factor (Regranex) on wound closure in genetically diabetic mice. J Burn Care Res 2006; 27(2):202-5.

12. Scherer SP, G; Mathews, J; Chan,R; Fiorina, P; Orgill, DP. Wound healing kinetics of genetically diabetic mouse. Wounds 2007.

13. Pietramaggiori G, Kaipainen A, Ho D, et al. Trehalose lyophilized platelets for wound healing. Wound Repair Regen 2007; 15(2):213-20.

14. Skalli O, Ropraz P, Trzeciak A, et al. A monoclonal antibody against alpha-smooth muscle actin: a new probe for smooth muscle differentiation. J. Cell Biol. 1986; 103(6 Pt 2):2787-96.

15. Hinz B, Mastrangelo D, Iselin CE, et al. Mechanical tension controls granulation tissue contractile activity and myofibroblast differentiation. Am J Pathol 2001; 159(3):1009-20.

16. Pietramaggiori G, Kaipainen A, Czeczuga JM, et al. Freeze-dried platelet-rich plasma shows beneficial healing properties in chronic wounds. Wound Repair Regen 2006; 14(5):573-80.

17. Modarressi A, Pietramaggiori G, Godbout C, et al. Hypoxia Impairs Skin Myofibroblast Differentiation and Function. J Invest Dermatol.

18. Hinz B, Celetta G, Tomasek JJ, et al. Alpha-smooth muscle actin expression upregulates fibroblast contractile activity. Mol Biol Cell 2001; 12(9):2730-41.

19. Goffin JM, Pittet P, Csucs G, et al. Focal adhesion size controls tension-dependent recruitment of alpha-smooth muscle actin to stress fibers. J Cell Biol 2006; 172(2):259-68.

20. Liu H, Chen B, Lilly B. Fibroblasts potentiate blood vessel formation partially through secreted factor TIMP-1. Angiogenesis 2008; 11(3):223-34. 
21. Bauer SM, Bauer RJ, Liu ZJ, et al. Vascular endothelial growth factor-C promotes vasculogenesis, angiogenesis, and collagen constriction in three-dimensional collagen gels. J Vasc Surg 2005; 41(4):699-707.

22. Hinz B. Formation and function of the myofibroblast during tissue repair. J Invest Dermatol 2007; 127(3):526-37.

23. Zhang Y, Tang H, Cai J, et al. Ovarian cancer-associated fibroblasts contribute to epithelial ovarian carcinoma metastasis by promoting angiogenesis, lymphangiogenesis and tumor cell invasion. Cancer Lett; 303(1):47-55.

24. Folkman J. Tumor angiogenesis: therapeutic implications. N Engl J Med 1971; 285(21):1182-6.

25. Italiano JE, Jr., Richardson JL, Patel-Hett S, et al. Angiogenesis is regulated by a novel mechanism: Pro- and anti-angiogenic proteins are organized into separate platelet \{alpha\}-granules and differentialy released. Blood 2007; 111(3):1227-33.

26. Kahn ML, Nakanishi-Matsui M, Shapiro MJ, et al. Protease-activated receptors 1 and 4 mediate activation of human platelets by thrombin. J Clin Invest 1999; 103(6):87987.

27. Bambace NM, Levis JE, Holmes CE. The effect of P2Y-mediated platelet activation on the release of VEGF and endostatin from platelets. Platelets 2010; 21(2):85-93. 


\section{FIGURES}

\section{Fig. 1. Diabetic mouse full thickness wound healing}

(Left column) Appearance of full thickness wounds 7 days post-wounding either healing spontaneously (NT), or treated with non-activated platelets $(\mathrm{P})$ or activated platelets $(\mathrm{aP})$. In the early phase of healing, non-activated platelets stimulated wound contraction (40\%, $\mathrm{p}<0.05)$ compared to activated platelets $(25 \%)$ and NT $(15 \%)$. Re-epithelialization (indicated by arrows) was similarly induced by both non-activated and activated platelets $(15 \%$ and $8 \%$, respectively) compared to non-treated wounds $(\mathrm{p}<0.05)$ where only scarce signs of reepithelialization were noted. Scale bar $0.5 \mathrm{~cm}$.

(Central column) PECAM-1 expression in healing wounds on day 7: increased levels of angiogenesis were found in platelets treated wounds compared to non-treated and activated platelet treatment. Scale bar 50 microns.

(Right Upper) In the later phase of healing, non-activated platelet treatment induced faster wound closure, reaching $90 \%$ wound closure within 15 days from wounding, while activated platelets treated wounds needed 26 days ( $p<0.01$ compared to platelets). Non-treated wounds needed more than 35 days to reach $90 \%$ wound closure.

(Right Middle) $\alpha$-SMA expression in granulation tissues on day 7 was augmented both in activated (3.8 fold) and non-activated platelet ( 6 fold) treated wounds compared to control wounds. Platelets induced a 1.5 fold higher $\alpha$-SMA expression in wounds over activated platelets treatment $(\mathrm{p}<0.05$, Lower central).

(Right Lower) Blood vessel density in 7-day old granulation tissue was 3.7 fold higher after platelet treatment than after treatment with activated platelets and 7.3 fold higher than untreated wounds.

$*<0.05, * *<0.01$, compared to untreated or activated platelet treated wounds. 
Fig. 2. Effects of platelet treatment on fibroblast cultures. Microscopic images show cultured human fibroblasts in three different experimental conditions: left column shows fibroblasts in control medium, central column shows fibroblasts treated with non-activated platelets $(\mathrm{P})$ and right column shows fibroblasts treated with activated platelets (aP). Three parameters are presented: fibroblast morphology, myofibroblast phenotype and contractile function.

(Left, Upper row) Morphology: 24 hours after seeding, fibroblasts showed a characteristic distribution when cultured with non-activated platelets $(\mathrm{P})$, forming extracellular areolar blood vessel like structures. Cells cultured in control media or activated platelets (aP) showed the usual pattern of cell adherence with random orientation.

(Right) High magnification (60x) of platelet-fibroblast co-cultures after 5 days immunostained with $\alpha$-SMA (green), vimentin (red) and nuclei (blue). Dendritic structures of fibroblasts are marked with white arrows. The connection of these structures formed a tubular like architecture.

(Middle row) Phenotype: Fluorescence microscopic images show $\alpha$-SMA stained myofibroblast (green) and cytoskeletal staining (F actin, red) and their respective nuclear staining (DAPI, blue) after 5 days of culture. Non-activated platelets induced increased levels of $\alpha$-SMA $(57.3 \%, \mathrm{p}<0.01)$ compared to control media $(21.5 \%)$ and activated platelets $(29.6 \%)$.

(Lower row) Function: The contractile activity of human dermal fibroblasts was assessed by the observation of soft substrate wrinkling induced by cells in control media, platelet and activated platelet treated cell cultures. Wrinkling was augmented after platelet $(35 \pm 3 \%$, $\mathrm{p}<0.05)$ compared to activated platelet treatment $(10 \pm 5 \%)$ and cells in control medium $(20 \pm 7 \%)$. Arrows indicated wrinkling of the substrate by contractile cells. 


\section{Fig. 3. In vitro 3D tissue defect assay.}

(Left) The center of a donut shaped fibroblast-populated collagen gel was filled with either non-activated or activated platelets.

Light microscopic pictures of the in vitro wound healing assay on day 7 with activated platelets (aP, Middle) or non-activated platelets (P, Right) are shown. Platelets allowed fibroblast migration into the tissue defect (arrows). When thrombin was added to platelets (aP), a clot formed in the centre, which prevented the migration of cells up to day 7 (no cells beyond the wound margin. Dotted line indicates the margin between original collagen defect and fibroblast seeded centre. Fibroblasts migrated into the platelets filled centre exhibited typical long dendritic extensions and areolar distribution.

Fig. 4. The effect of physiological and unspecific activation of platelets on wound healing.

(Left) Non-activated platelets represent a drug delivery system controlled by specific release of pro-angiogenic and anti-angiogenic factors.

(Right) Activated platelets release high doses of both stimulatory and inhibitory factors, with counter-regulatory effect. 

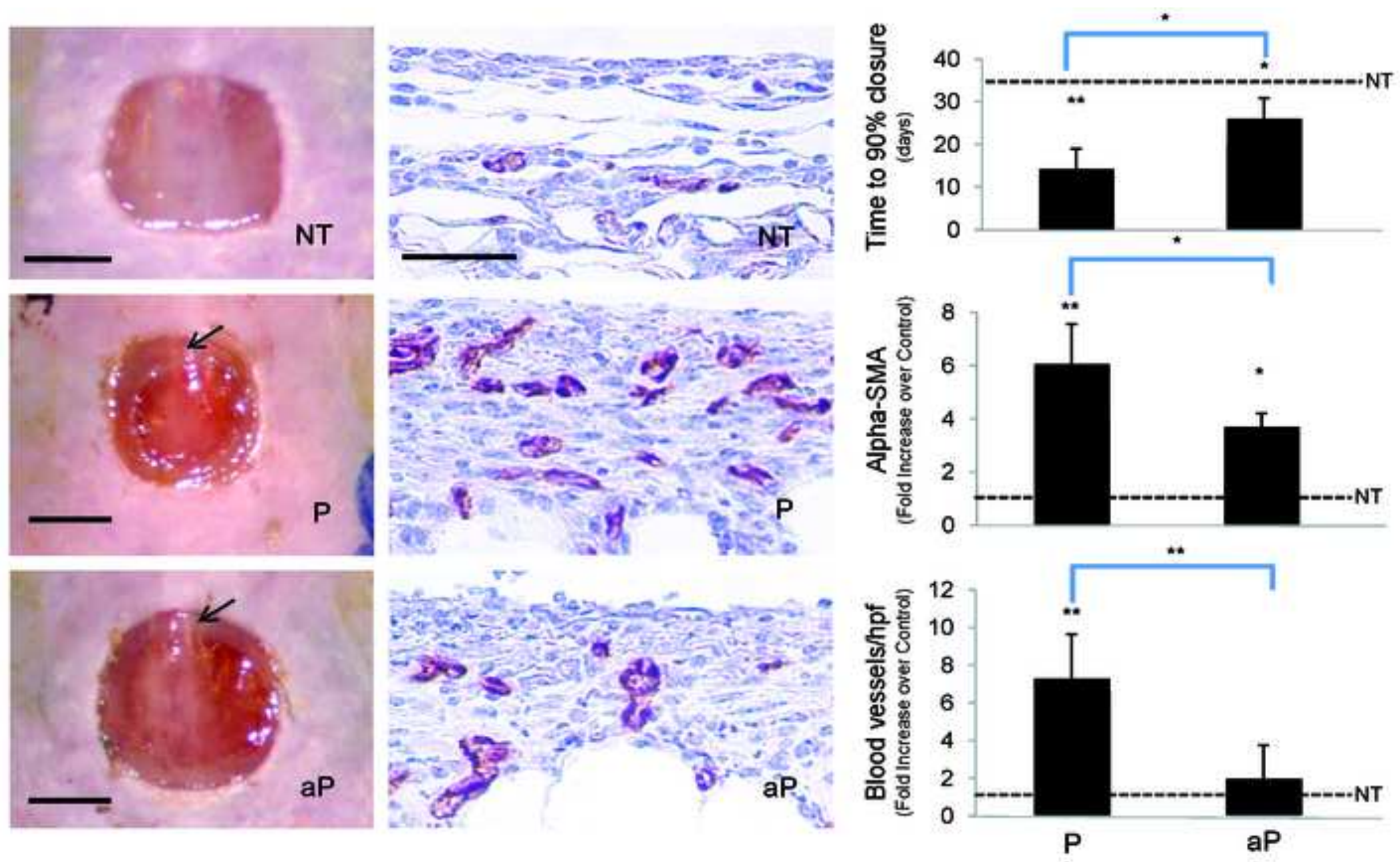
Click here to download high resolution image

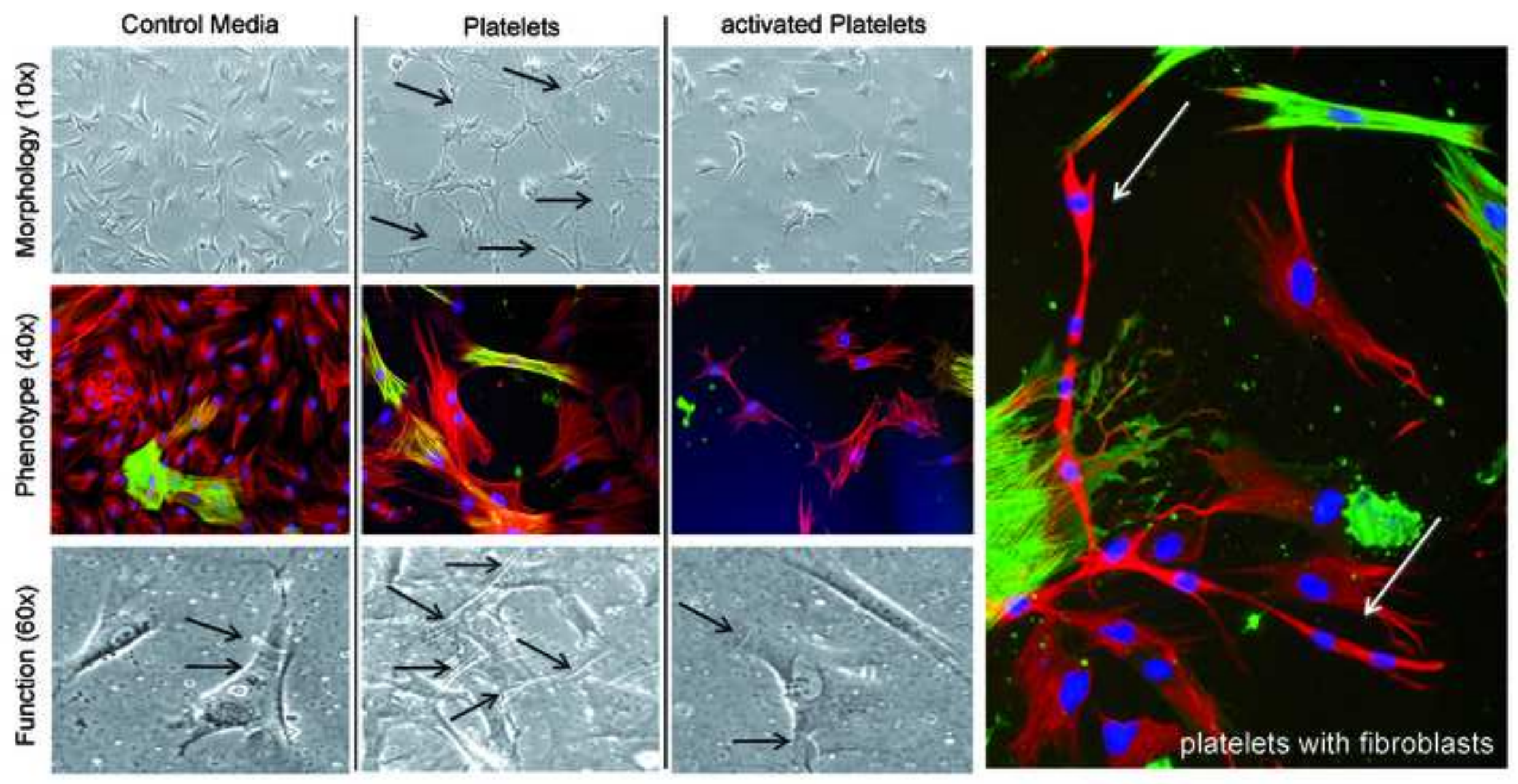




\section{igure 3}

Click here to download high resolution image

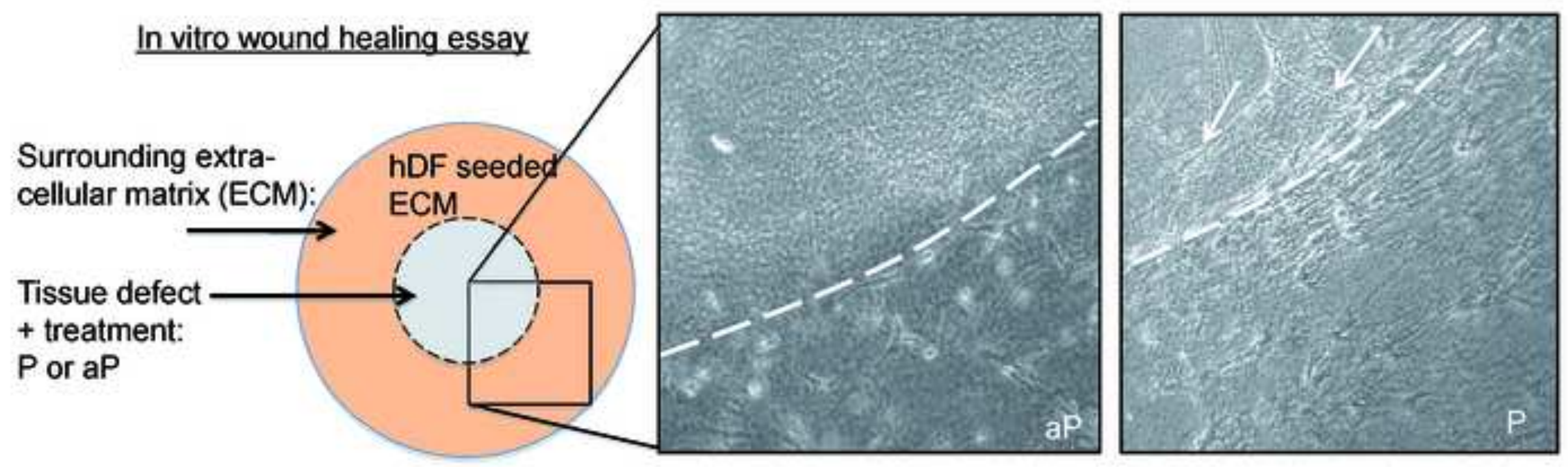


Non activated functional platelet $(P)$

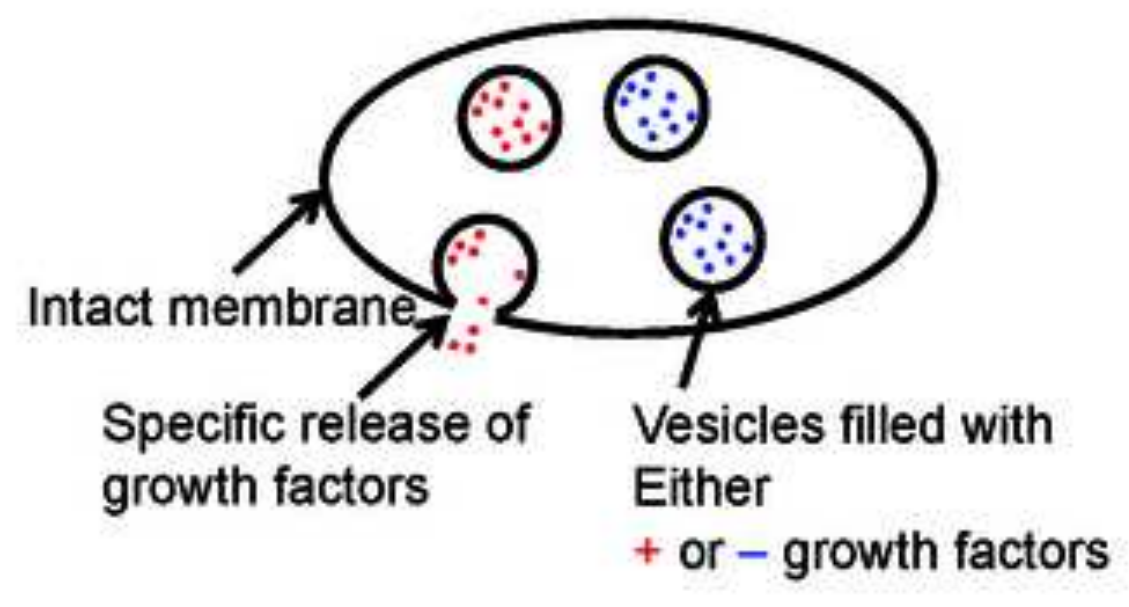

Platelet after terminal degranulation with Thrombin (aP)

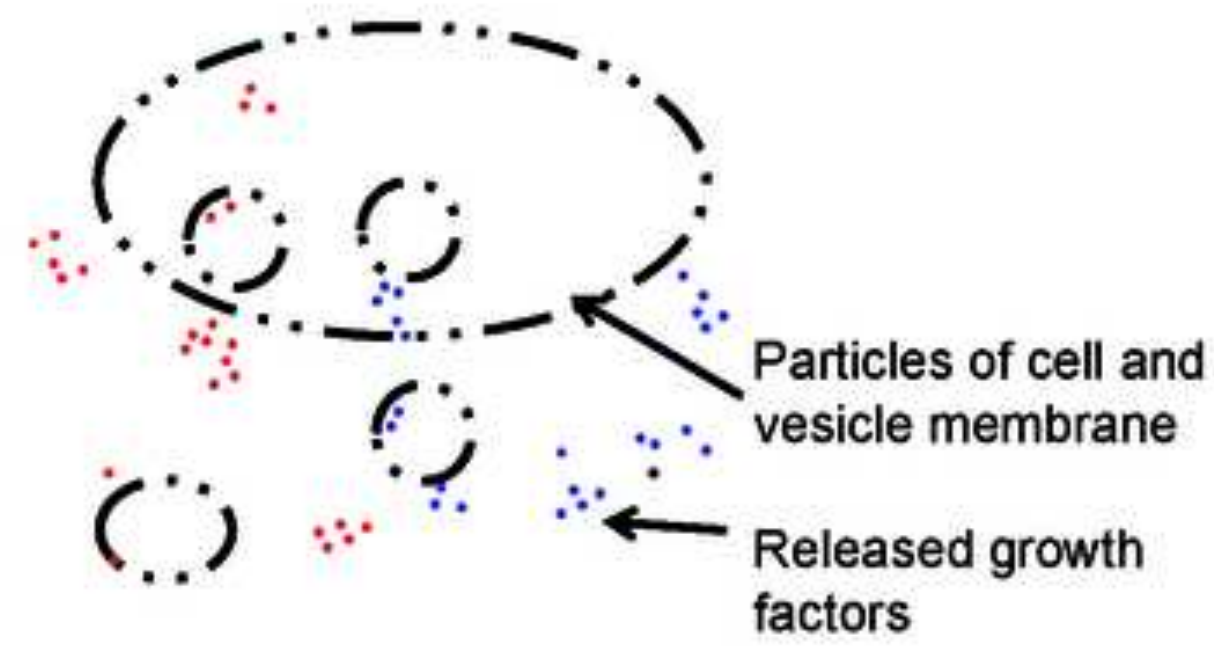


Lausanne, April $29^{\text {th }}, 2010$

\section{Editor}

Rod J. Rohrich, M.D.

Editor in chief Plastic and Reconstructive Surgery Journal

Dear Dr. Rohrich,

We would like to submit a manuscript entitled: "Non-activated versus thrombinactivated platelets on wound healing and fibroblast-to-myofibroblast differentiation in vivo and in vitro" to the journal Plastic and Reconstructive Surgery.

It has been assumed that activating platelets before application on wounds is the best way to exploit platelet healing potential.

We found that non-pre-activated platelets have a superior ability to improve healing by inducing more efficient wound closure than thrombin activated platelets in vitro and in a diabetic mouse model.

The manuscript has not been published previously and is not being considered concurrently by another journal. All authors and acknowledged contributors have read and approved the manuscript. We suggest drs. Eppley Barry L (11725 N Illinois St Ste 140 Carmel, Indiana 46032) and Klement L Giannoula (Center for Cancer system Biology, Caritas St. Elizabeth's Health Center 736 Cambridge Street, CBR 404 Boston, MA 02135) as reviewers due to their clinical and basic science contributions in the fields of platelet biology.

Thank you very much for your kind consideration,

Giorgio Pietramaggiori MD, PhD

Division of Plastic Surgery

Centre hospitalier universitaire vaudois

Rue du Bugnon 21

1011 Lausanne

0213141111 
Author Forms (Prior Publication Certification, Copyright Form

Author Forms (Prior Publication Certification, Copyright Form, Conflict of Interest Disclosure)

)

Author Forms (P

(n)

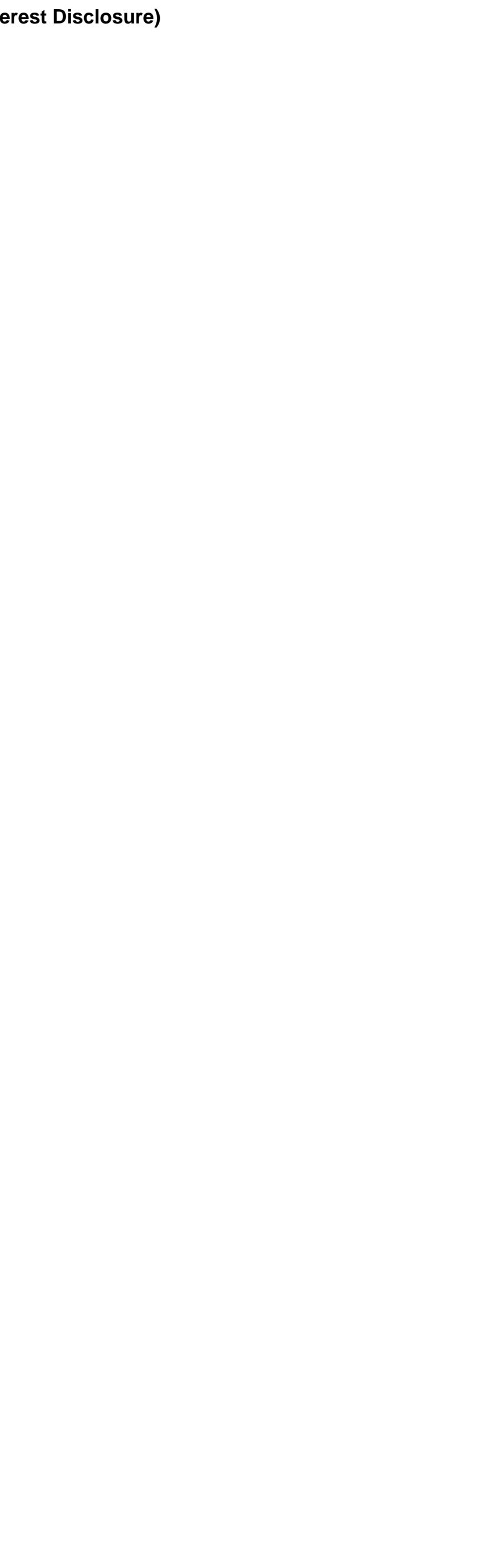


Form 3. Conflict of Interest Disclosure stakement by an Author of a manuscript submitted to Plastic and Reconstructive Surgery

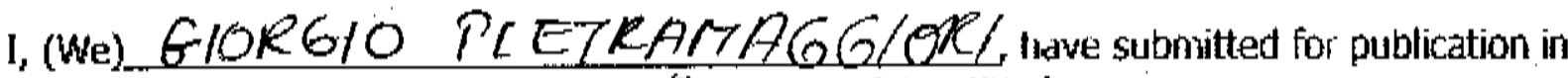
Plastic and Reconstructive Surgervis a manuscript entitled:

Comparis on between non-achivated and thrombin-achived platelets to inderce wound healing and tibroblast-myofibroblast hansdifteren-

tiation : in vivo-is witro

No financial support or benefits have been receivol by me or any cis-author, by any member of my (our) comparion inmediale family of any individual or entity with whom or with which I (we) have a selationship from any commercial source shich is retated directly or indirectly to the stientific work which is reposted on sn the article except as destribed below.

\{t (we) understand an cxample of such a financial interest would be $\mathrm{J}$ consulting relationship or stock interest in any business entity which is included in the subiect mater of the manuscript or which sells a product relating to the subject matter of ure rumuscript.

In addition to filling oul ind signing this declaration, 1 actwowledge that I (we) whis mate womplete disclosure in the mumuscripi itselfs stating all sources of funds that tave supporled this work and also a statenent of nnincial inleresd, if any. Each auther on the mandscript has disclosed any commercial assictation or fiuancial disclosure that might pest a connict of interest with information presented in this manuscript. If the aw homs hove mo finenctat interest of commercial association with any of the subject muter or products mewioned in ow mamnseript, that too will be indicated. 1 (we) hereby agree that such

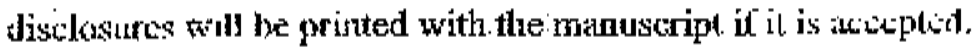

Furhermore, 1 (we) understand that potential sanctions may be imposed ty lasstie and Reconstractive Surcery for wiolation of this complete disclosure policy. I (we) undersland that potential disciplinary antions may include wanning letlers, fefusal to publish an article in questron, retraction of a published paper, notification to our primaly instintion, andor exchusion from publication in Plastic and Reconsinas five Surgery for a specified time frame.

The conespondiog anthor has the obligation of having any and all wo-iuthors sign this form and date his or her xignithre.
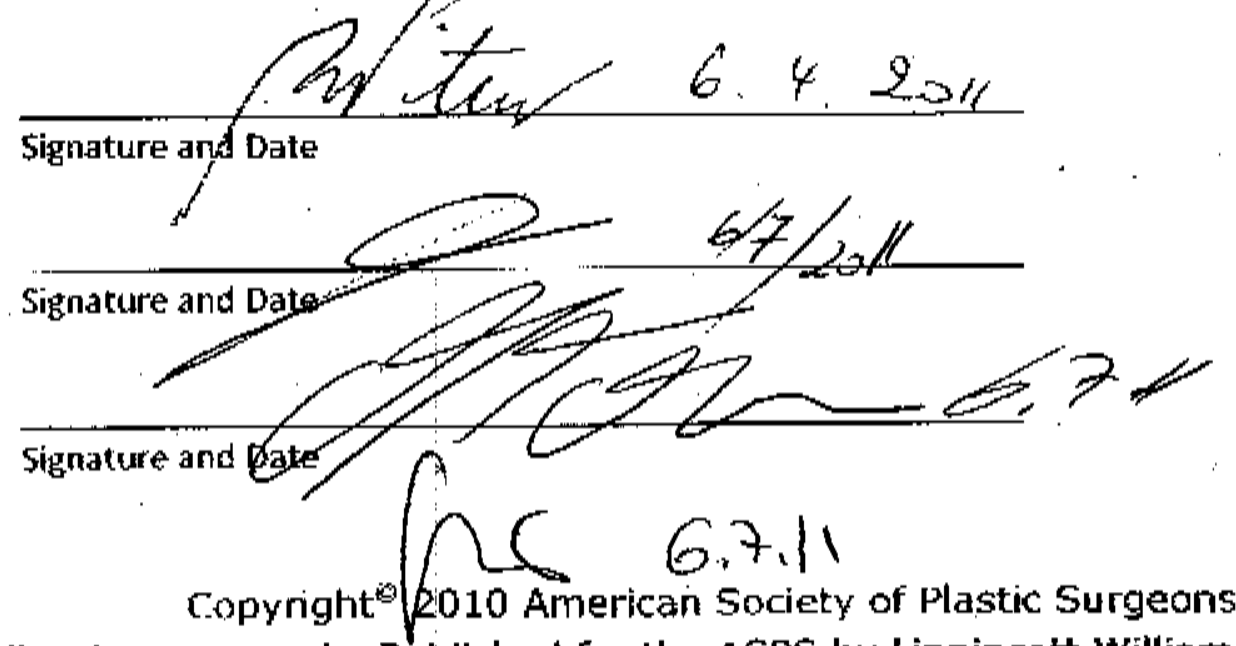

All rughts reserved - Published for the ASPS by Lippincott Williams \& Walkıns

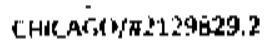

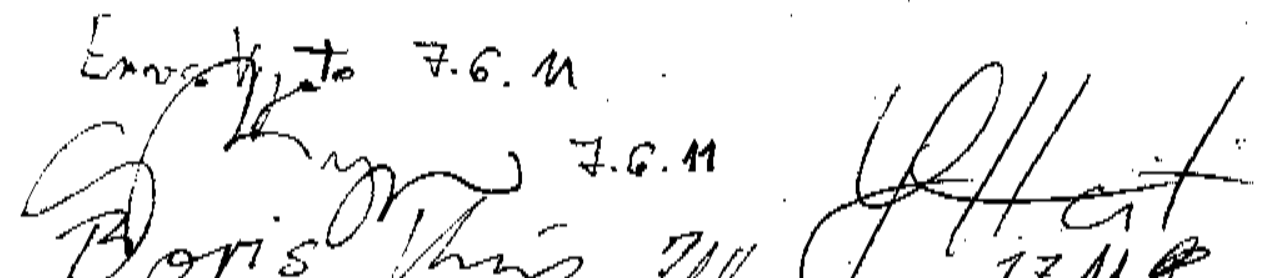


